K. BOGDAN

KODAI MATH. J.

19 (1996), 7-16

\title{
ON THE ZEROS OF FUNCTIONS WITH FINITE DIRICHLET INTEGRAL
}

\author{
KRZYSZTOF BOGDAN*
}

\begin{abstract}
We characterize subsets $A$ of the unit disc in the plane such that every Blaschke sequence with elements in $A$ is the sequence of zeros of an analytic function with finite Dirichlet integral.

Also, assuming that arguments are uniformly distributed independent random variables, we characterize the moduli of sequences which are almost surely zero sequences for the same function family.
\end{abstract}

\section{Introduction}

We consider the Dirichlet space $\mathscr{D}=\mathscr{D}(\Delta)$ of functions $f$ analytic on $\Delta=$ $\{z \in C:|z|<1\}$ with finite Dirichlet integral

$$
\|f\|^{2}=\int_{\Delta}\left|f^{\prime}(z)\right|^{2} d m
$$

By $m$ we denote the planar measure on $\Delta$. A simple computation shows that if $f(z)=\sum_{n=0}^{\infty} a_{n} z^{n}$ is analytic in $\Delta$, then

$$
\|f\|^{2}=\pi \sum_{n=0}^{\infty} n\left|a_{n}\right|^{2},
$$

and so $\mathscr{D}$ is contained in the Hardy space $H^{2}$. In particular, this implies that every sequence $\left\{z_{n}\right\}$ of zeros (counting multiplicities) of a function $f \in \mathscr{D}, f \neq 0$, (i.e. a zero sequence for $\mathscr{D})$ necessarily satisfies the Blaschke condition

$$
\Sigma\left(1-\left|z_{n}\right|\right)<\infty \text {. }
$$

It is well known that (3) and the condition

$$
-\int_{-\pi}^{\pi} \log \operatorname{dist}\left(Z, e^{i t}\right) d t<\infty,
$$

1991 Mathematics Subject Classification, Primary $30 \mathrm{C} 15$.

Key words and phrases. Analytic functions, zero sets, Dirichlet space.

* Supported by the grant KBN 211599101.

Received June 1, 1994 ; revised April 21, 1995. 
characterize the sequences of zeros (counting multiplicities) $Z=\left\{z_{n}\right\}$ of functions in the classes $\operatorname{Lip}_{\alpha}(\Delta), \alpha>0$. (As usual, dist above denotes the Euclidean distance on the plane.) Moreover, (3) and (4) imply that there exists an analytic function $f \neq \equiv 0$ having $Z$ as zeros, with all its derivatives bounded in $\Delta$. This result was proved in [10] and, indepedently, in [8] (see also [4] and [7]). Consequenly these conditions are sufficient for the existence of such a function $f \in \mathscr{D}$. A necessary condition cannot be expressed in such a way because there exists a zero sequence for $\mathscr{D}$, which is at the zero distance from every point of the boundary $\boldsymbol{T}=\bar{\Delta} \backslash \Delta$ of $\Delta$, see below. (It was proved in [11] that for $f \neq 0$, $f^{\prime} \in H^{1}$, (4) necessarily holds ; see also [5].)

Definition 1. We call $A \subset \Delta$ a Blaschke set for $\mathscr{D}$ if every Blaschke sequence with elements in $A$ is a zero sequence for $\mathscr{D}$.

Results proved in [4] and [7] show that a radial subset $Z$ of $\Delta$ (i.e. the union of radii) satisfies (4) if and only if every Blaschke sequence in $Z$ is a zero sequence for $\mathscr{D}$. We are strenghtening this assertion by the following theorem.

THEOREM 1. A set $A \subset \Delta$ is Blaschke for $\mathscr{D}$ if and only if

$$
-\int_{-\pi}^{\pi} \log \operatorname{dist}\left(A, e^{i t}\right) d t<\infty .
$$

Moreover, if (5) holds then there is an outer function $F \in \mathscr{D}$ with the property that $B F \in \mathscr{D}$ for every Blaschke product $B$ with zeros in $A$.

Most of this paper is devoted to the proof of this result. The main tool we use is the formula for the Dirichlet integral given by Carleson in [2]:

$$
\begin{aligned}
\|f\|^{2}= & \frac{1}{2} \int_{-\pi}^{\pi} \sum \frac{1-\left|z_{n}\right|^{2}}{\left|e^{i t}-z_{n}\right|^{2}}\left|f\left(e^{i t}\right)\right|^{2} d t+\frac{1}{4} \int_{-\pi}^{\pi} \int_{-\pi}^{\pi} \frac{\left|f\left(e^{i t}\right)\right|^{2} d s(\varphi)}{\sin ^{2} \frac{t-\varphi}{2}} d t \\
& +\frac{1}{8 \pi} \int_{0}^{\pi} \frac{d t}{\sin ^{2} \frac{t}{2}} \int_{-\pi}^{\pi}\left(\left|f\left(e^{i(t+x)}\right)\right|^{2}-\left|f\left(e^{i x}\right)\right|^{2}\right) \\
& \times\left(\log \left|f\left(e^{\imath(t+x)}\right)\right|-\log \left|f\left(e^{i x}\right)\right|\right) d x,
\end{aligned}
$$

where

$$
\begin{aligned}
f(z)= & C \Pi \frac{\bar{z}_{n}}{\left|z_{n}\right|} \frac{z_{n}-z}{1-z \bar{z}_{n}} \exp \left(-\int_{\pi}^{\pi} \frac{e^{i t}+z}{e^{i t}-z} d s(t)\right) \\
& \times \exp \left(\frac{1}{2 \pi} \int_{\pi}^{\pi} \frac{e^{i t}+z}{e^{i t}-z} \log \left|f\left(e^{i t}\right)\right| d t\right) .
\end{aligned}
$$

Here $\left\{z_{n}\right\}$ denotes the sequence of zeros (counting multiplicities) of $f$; $s$ is the singular measure of $f$, and $C$ is a constant of modulus 1 . Note that if $f=$ 
$C B S F$ is the above canonical factorization of $f$ into a constant multiple of the Blaschke product, the singular factor and the outer part respectively, then (6) yields

$$
\|f\|^{2}=\left(\|B F\|^{2}-\|F\|^{2}\right)+\left(\|S F\|^{2}-\|F\|^{2}\right)+\|F\|^{2},
$$

each summand being nonnegative. The Jensen inequality and (6) yield the following important necessary condition on zero sequences $\left\{z_{n}\right\}$ for $\mathscr{D}$ :

$$
\int_{-\pi}^{\pi} \log \left(\sum \frac{1-\left|z_{n}\right|^{2}}{\left|e^{i t}-z_{n}\right|^{2}}\right) d t<\infty,
$$

see [3]. A much stronger condition

$$
\sum \frac{1}{\log _{\frac{1-r_{n}}{1-n}}}<\infty,
$$

where $r_{n}=\left|z_{n}\right|$, as shown by Shapiro and Shields in [9], is sufficient for $\left\{z_{n}\right\}$ to be a zero sequence for $\mathscr{D}$. Conversely, it was proved in [6] that if a sequence of numbers $r_{n} \in(0,1), n=1,2, \cdots$, does not satisfy (9), then there is a sequence of numbers $\theta_{n} \in(-\pi, \pi]$ with the property that $z_{n}=r_{n} e^{i \theta_{n}}$, is not a zero sequence for $\mathscr{D}$. Now, we regard each $\theta_{n}$ above as value of a randon variable $\Theta_{n}$. In this probabilistic setting we have the following result.

THEOREM 2. Let $\left\{\Theta_{n}\right\}$ be a sequence of independent random variables uniformly distributed on $(-\pi, \pi]$; let $r_{n} \in(0,1)$ and $Z_{n}=r_{n} e^{i \theta_{n}}, n=1,2, \cdots$. If (9) holds then each realization of $\left\{Z_{n}\right\}$ is a zero sequence for $\mathscr{D}$. Conversely if (9) fails to hold then almost surely $\left\{Z_{n}\right\}$ is a not a zero sequence for $\mathscr{D}$.

\section{Proof of Theorem 1}

Definition 2. For $z=r e^{\imath \varphi} \in \Delta$, we write $S_{z}=\left\{w=\rho e^{\imath \sigma} \in \Delta: \rho \leqq r,|\varphi-\sigma|<\right.$ $1 / 2(1-\rho)\}$, and we define $\tilde{A}=\cup_{z \in A} S_{z}$, for $A \subset \Delta$.

We need the following estimate

$$
C_{1}\left\{(1-r)^{2}+\varphi^{2}\right\} \leqq\left|e^{\imath \varphi}-r\right|^{2} \leqq(1-r)^{2}+\varphi^{2}, r \in[0,1), \varphi \in(-\pi, \pi],
$$

(some $C_{1}>0$ ) which is an easy consequence of

$$
\left|e^{\imath \varphi}-r\right|^{2}=1-2 r \cos \varphi+r^{2}=(1-r)^{2}+r\left(2 \sin \frac{\varphi}{2}\right)^{2} .
$$

If $z=r e^{\imath \varphi} \in \Delta$, we write, as usual, $P_{z}$ for the Poisson kernel

$$
P_{z}\left(e^{i t}\right)=\frac{1-|z|^{2}}{\left|e^{i t}-z\right|^{2}}=\frac{1-r^{2}}{1-2 r \cos (t-\varphi)+r^{2}}, \quad t \in(-\pi, \pi] .
$$


LEMMA 1. There is a constant $C_{2}<\infty$ such that for all $z \in \Delta, w \in S_{z}$

$$
P_{w}\left(e^{i t}\right) \leqq C_{2} \frac{1-|w|}{1-|z|} P_{z}\left(e^{i t}\right), \quad t \in(-\pi, \pi] .
$$

The derivation of (11) from (10) is straightforward and we skip it here. We observe that (11) is equivalent to $\left|e^{i t}-z\right|^{2} \leqq C_{2}(1+|z|) /(1+|w|)\left|e^{i t}-w\right|^{2}, t \in$ $(-\pi, \pi]$. It follows that $\operatorname{dist}\left(\tilde{A}, e^{i t}\right) \leqq \operatorname{dist}\left(A, e^{i t}\right) \leqq \sqrt{2 C_{2}} \operatorname{dist}\left(\tilde{A}, e^{i t}\right), A \subset \Delta$, and

$$
-\int_{-\pi}^{\pi} \log \operatorname{dist}\left(A, e^{i t}\right) d t<\infty \text { if and only if }-\int_{-\pi}^{\pi} \log \operatorname{dist}\left(\tilde{A}, e^{i t}\right) d t<\infty .
$$

LeMma 2. A set $A \subset \Delta$ is Blaschke if and only if $\tilde{A}$ is Blaschke.

Proof. If $\tilde{A}$ is a Blaschke set then $A$ is so, since $A \subset \tilde{A}$.

Now assume that $A$ is a Blaschke set. Let $\left\{\alpha_{n}\right\}$ be a sequence of elements of $\tilde{A}$ with $\Sigma\left(1-\left|\alpha_{n}\right|\right)<\infty$. Let $\left\{z_{n}\right\}$ be a sequence of points in $A$ such that $\alpha_{n} \in S_{z_{n}}$ for every $n$. Also, let $p_{n}=\left[\left(1-\left|\alpha_{n}\right|\right) /\left(1-\left|z_{n}\right|\right)\right]$ be the integer part of $\left(1-\left|\alpha_{n}\right|\right) /\left(1-\left|z_{n}\right|\right)$. Notice that

$$
\frac{1}{2} \frac{1-\left|\alpha_{n}\right|}{1-\left|z_{n}\right|}<p_{n} \leqq \frac{1-\left|\alpha_{n}\right|}{1-\left|z_{n}\right|},
$$

because $\left(1-\left|\alpha_{n}\right|\right) /\left(1-\left|z_{n}\right|\right) \geqq 1$. We construct an auxiliary sequence $Z$, replacing each $\alpha_{n}$ in the sequence $\left\{\alpha_{n}\right\}$ by $p_{n}$ occurrences of $z_{n}$. By (13), $\sum p_{n}\left(1-\left|z_{n}\right|\right)$ $\leqq \Sigma\left(1-\left|\alpha_{n}\right|\right)$, and so $Z$ satisfies the Blaschke condition. Let $B=B(Z)$ be the Blaschke product for $Z$. Our assumption on $A$ implies that there is an outer function $F \in \mathscr{D}$ (see (7)) such that $B F \in \mathscr{D}$. In particular, by (6),

$$
\frac{1}{2} \sum_{n} p_{n} \int_{-\pi}^{\pi} \frac{1-\left|z_{n}\right|^{2}}{\left|e^{i t}-z_{n}\right|^{2}}\left|F\left(e^{i t}\right)\right|^{2} d t<\infty .
$$

Let $J$ be the Blaschke product for $\left\{\alpha_{n}\right\}$. Applying (6) gives

$$
\|J F\|^{2}=\|F\|^{2}+\frac{1}{2} \int_{-\pi}^{\pi} \sum_{n} \frac{1-\left|\alpha_{n}\right|^{2}}{\left|e^{i t}-\alpha_{n}\right|^{2}}\left|F\left(e^{i t}\right)\right|^{2} d t .
$$

We claim that the second term is finite. Indeed, by Lemma 1 and (13), $\left(1-\left|\alpha_{n}\right|^{2}\right) /\left|e^{i t}-\alpha_{n}\right|^{2} \leqq 2 C_{2} p_{n}\left(1-\left|z_{n}\right|^{2}\right) /\left|e^{i t}-z_{n}\right|^{2}$. This combined with (14), proves the assertion.

Definition 3. Fix $A \subset \Delta$ and set

$$
J_{n}=\left\{t \in(-\pi, \pi]:\left(1-2^{-n}\right) e^{i t} \in \tilde{A}\right\}, \quad n=1,2, \cdots .
$$

We state below several simple properties of the sets $J_{n}$.

$J_{n}$ is a finite union of disjoint open intervals $I$ of length $|I| \geqq 2^{-n}$,

$$
J_{1} \supset J_{2} \supset J_{3} \supset \cdots,
$$




$$
\begin{gathered}
\sum_{n=1}^{\infty}\left|J_{n}\right|=\sum_{n=1}^{\infty} n\left|J_{n} \backslash J_{n+1}\right|+\infty \cdot \lim _{n \rightarrow \infty}\left|J_{n}\right|, \\
-\int_{-\pi}^{\pi} \log \operatorname{dist}\left(A, e^{i t}\right) d t<\infty \text { if and only if } \sum_{n=1}^{\infty}\left|J_{n}\right|<\infty,
\end{gathered}
$$

with the usual convention $\infty \cdot 0=0$ in (17).

Let us explain why (18) holds. Consider the radial distance $d$ :

$$
d\left(\tilde{A}, e^{i t}\right)=\inf \left\{\kappa:(1-\kappa) e^{i t} \in \tilde{A}\right\}, \quad t \in(-\pi, \pi] .
$$

We easily observe that there is a constant $C_{3}<\infty$ such that

$$
\operatorname{dist}\left(\tilde{A}, e^{\imath t}\right) \leqq d\left(\tilde{A}, e^{i t}\right) \leqq C_{3} \operatorname{dist}\left(\tilde{A}, e^{\imath t}\right), \quad t \in(-\pi, \pi] .
$$

For $A \neq \emptyset$, the definition of $d$ and (17) yield

$$
\begin{aligned}
\sum_{n=1}^{\infty}\left|J_{n}\right| & =\sum_{n=1}^{\infty} n\left|J_{n} \backslash J_{n+1}\right|+\infty \lim _{n \rightarrow \infty}\left|J_{n}\right| \leqq-\int_{-\pi}^{\pi} \log _{2} d\left(\tilde{A}, e^{i t}(d t\right. \\
& \leqq 2 \pi+\sum_{n=1}^{\infty}(n+1)\left|J_{n} \backslash J_{n+1}\right|+\infty \lim _{n \rightarrow \infty}\left|J_{n}\right| \leqq \sum_{n=1}^{\infty}\left|J_{n}\right|+4 \pi
\end{aligned}
$$

Hence by (19), (18) is proved.

Definition 4. We write $R_{n}$ for the difference $J_{n} \backslash J_{n+1}$ and $R_{n}^{L}$ for the union of all the connected components $I$ of $R_{n}$ satisfying $|I|>2^{-(n+3)}$.

It is clear that $R_{n}$ is a finite union of pairwise disjoint intervals and points.

LEMMA 3. We have $\sum_{n=1}^{\infty} n\left|R_{n}^{L}\right|<\infty$ if and only if $\sum_{n=1}^{\infty} n\left|R_{n}\right|<\infty$.

Proof. Let $R_{n}^{s}$ be the union of all those (connected) components of $R_{n}$ which have length less than or equal to $2^{-(n+3)}$ (so that $R_{n}=R_{n}^{L} \cup R_{n}^{s}$ ) and let $c_{n}$ denote the number of such components. Fix a component $I$ of $R_{n}^{s}$. Let $P$ be the first endpoint of $I$. (We adopt the natural orientation of $\boldsymbol{T} \sim(-\pi, \pi]$.) By definition $I \cap J_{n+1}=\emptyset$. Also, $P$ is in the closure of $J_{n+1}$ since otherwise $|I|$ $\geqq 2^{-(n+2)}$ (a sketch of $\tilde{A}$ in polar coordinates may be useful). Note that $P$ is the (second) endpoint of a component, say $I_{P}$, of $J_{n+1}$, and, by (15), $\left|I_{P}\right| \geqq$ $2^{-(n+1)}$. While passing from $J_{n+1}$ to $J_{n+2}$, each components of $J_{n+1}$ gets smaller by at least $2 \cdot 2^{-(n+3)}$ i. e. $\left|I_{P} \backslash J_{n+2}\right| \geqq 2 \cdot 2^{-(n+3)}$, therefore $\left|R_{n+1}\right|=\left|J_{n+1} \backslash J_{n+2}\right| \geqq$ $2^{-(n+2)} c_{n}$, and so $\left|R_{n}^{s}\right| \leqq 2^{-(n+3)} c_{n} \leqq 1 / 2\left|R_{n+1}\right|$. Then

$$
\sum_{n=1}^{\infty} n\left|R_{n}\right|=\sum_{n=1}^{\infty} n\left|R_{n}^{s}\right|+\sum_{n=1}^{\infty} n\left|R_{n}^{L}\right| \leqq \frac{1}{2} \sum_{n=1}^{\infty} n\left|R_{n+1}\right|+\sum_{n=1}^{\infty} n\left|R_{n}^{L}\right|,
$$

which yields 


$$
\begin{aligned}
\sum_{n=1}^{\infty} n\left|R_{n}^{L}\right| & \geqq \sum_{n=1}^{\infty} n\left|R_{n}\right|-\frac{1}{2} \sum_{n=1}^{\infty}(n+1)\left|R_{n+1}\right|+\frac{1}{2} \sum_{n=1}^{\infty}\left|R_{n+1}\right| \\
& =\frac{1}{2} \sum_{n=1}^{\infty} n\left|R_{n}\right|+\frac{1}{2} \sum_{n=1}^{\infty}\left|R_{n}\right|,
\end{aligned}
$$

and the desired conclusion follows.

LEMMA 4. If $\tilde{A}$ is a Blaschke set then $-\int_{-\pi}^{\pi} \log \operatorname{dist}\left(\tilde{A}, e^{i t}\right) d t<\infty$.

Proof. Let $\tilde{A}$ be a Blaschke set. We first prove the lemma under the assumption $\lim _{n \rightarrow \infty}\left|J_{n}\right|=0$. For $n=1,2, \cdots$, consider the finite sequence $Z_{n}$ of all those points $z \in \Delta$, which are of the form $z=\left(1-2^{-n}\right) \exp \left(i k 2^{-(n+3)}\right), k=0,1, \cdots$, $2^{n+3}-1$, and such that $k 2^{-(n+3)} \in R_{n}^{L}$. Let $M_{n}$ be the number of those points. Also, for each component $I$ of $K_{n}^{L}$, let $c_{I}$ be the number of elements of $Z_{n}$ with $k 2^{-(n+3)} \in I$ (so that $M_{n}=\sum_{I} c_{I}$ ). Observe that, by Definition $4, c_{I} \geqq 1$, and, moreover, $(1 / 2)|I| 2^{n+3}<c_{I} \leqq 2|I| 2^{n+3}$, which yields

$$
\frac{1}{2}\left|R_{n}^{L}\right| 2^{n+3} \leqq M_{n} \leqq 2\left|R_{n}^{L}\right| 2^{n+3} .
$$

Let $Z=\left\{z_{n}\right\}$ denote the sequence obtained by putting all the sequences $Z_{n}$ together. We claim that $Z$ satisfies (3). Indeed,

$$
\sum_{n=1}^{\infty} \sum_{Z_{n}}(1-|z|)=\sum_{n=1}^{\infty} M_{n} 2^{-n} \leqq \sum_{n=1}^{\infty} 2\left|R_{n}^{L}\right| 2^{n+3} 2^{-n} \leqq 32 \pi .
$$

(If $S$ is a sequence, $\Sigma_{s}$ is the sum taken over its elements.) Again, let $I$ be a component of $R_{n}^{L}$. For each $t \in I$, there is an element $z=r e^{2 \varphi}$ of $Z_{n}$ such that $|\varphi-t|<2^{-(n+3)}$. We apply (10) to get

$$
\frac{1-|z|^{2}}{\left|e^{i t}-z\right|^{2}} \geqq \frac{2^{-n}\left(1+2^{-n}\right)}{\left(2^{-n}\right)^{2}+\left(2^{-(n+3)}\right)^{2}} \geqq 2^{n-1} .
$$

By the assumption on $\tilde{A}, Z$ is a zero sequence for the class $\mathscr{D}$, and the same is true for the sequence $Z^{\prime}$ obtained by adding $z=0$ to $Z$. Then (8) implies

$$
\begin{aligned}
\infty & >\int_{-\pi}^{\pi} \log \left(\sum_{Z^{\prime}} \frac{1-|z|^{2}}{\left|e^{i t}-z\right|^{2}}\right) d t \geqq \sum_{n=1}^{\infty} \int_{R_{n}^{L}} \log 2^{n-1} d t \\
& =\log 2 \sum_{n=1}^{\infty}(n-1)\left|R_{n}^{L}\right| \geqq \log 2 \sum_{n=1}^{\infty} n\left|R_{n}^{L}\right|-2 \pi \log 2 .
\end{aligned}
$$

By Lemma 3, (17) and (18), we get the desired conclusion. The proof will be complete when we show that if $\lim _{n \rightarrow \infty}\left|J_{n}\right| \neq 0$, then $\tilde{A}$ is not a Blaschke set. Assume that the intersection $J_{\infty}=\bigcap_{n=1}^{\infty} J_{n}$ is of positive Lebesgue measure. We claim that there is a sequence $\left\{\varepsilon_{n}\right\}$ of positive numbers satisfying

$$
\sum \varepsilon_{n} \leqq\left|J_{\infty}\right|, \quad \sum \varepsilon_{n} \log \frac{1}{\varepsilon_{n}}=\infty,
$$


and a collection $\left\{I_{n}\right\}$ of disjoint intervals in $(-\pi, \pi]$ such that

$$
\left|I_{n}\right|=\varepsilon_{n}, \quad I_{n} \cap J_{\infty} \neq \emptyset, \quad n=1,2, \cdots .
$$

We do not give detailed construction here. For $\varphi_{n} \in I_{n} \cap J_{\infty}$, we put $z_{n}=\left(1-\varepsilon_{n}\right) e^{\imath \varphi_{n}}$, to obtain a Blaschke sequence in $\tilde{A}$ for which, by (21) and the method discussed above, (8) fails to hold. It follows that $\tilde{A}$ is not a Blaschke set, which completes the proof.

Let us remark that the idea of using (8) in investigation of zeros of functions in the class $\mathscr{D}$ is due to Carleson (see [3], [4]).

Lemma 5. If $1>\varepsilon>0$ and $g \in C^{1+\varepsilon}(\boldsymbol{T})$ is nonnegative, then the Poisson integral of $g$ is $O(1-|z|)$ on the set $W=\left\{z=r e^{\imath \varphi} \in \Delta: 1-r \geqq g(\varphi)\right\}$.

Proof. Let $0<\varepsilon<1$ and $g \in C^{1+\varepsilon}(\boldsymbol{T})$, meaning that $g \in C^{1}(\boldsymbol{T})$ and $g^{\prime} \in \operatorname{Lip}_{\varepsilon}(\boldsymbol{T})$. By Taylor's formula, (10) and a simple change of variables we get, for $z=$ $r e^{\imath \varphi} \in \Delta$

$$
\begin{aligned}
P_{z}[g] & =\frac{1}{2 \pi} \int_{-\pi}^{\pi} \frac{1-r^{2}}{1-2 r \cos u+r^{2}} g(\varphi-u) d u \\
& =\frac{1}{2 \pi} \int_{-\pi}^{\pi} \frac{1-r^{2}}{1-2 r \cos u+r^{2}}\left[g(\varphi)-u g^{\prime}(\varphi)+O\left(u^{1+\varepsilon}\right)\right] d u \\
& =g(\varphi)+O\left(\int_{-\pi}^{\pi} \frac{(1-r) u^{1+\varepsilon}}{(1-r)^{2}+u^{2}} d u\right)=g(\varphi)+O(1-r)=O(1-r),
\end{aligned}
$$

provided $z \in W$. This completes the proof.

Proof of Theorem 1. If $A$ is a Blaschke set then Lemmas 2, 4 and (12) clearly show that (5) holds.

Now assume that (5) is satisfied. Let $Z$ be a Blaschke sequence with elements in $A$. Then, of course, we have (4), and, by the result in [10], there is an analytic function $f \in C^{\infty}(\bar{\Delta})$, vanishing precisely on $Z$. Clearly $f \in \mathscr{D}$.

To prove the second part of the theorem, we proceed as follows. Let $A$ be a Blaschke set. Suppose that there exists an outer function $F \in \mathscr{D}$ such that

$$
P_{z}\left[\left|F\left(e^{i t}\right)\right|^{2}\right]=O(1-|z|) \quad \text { on } A \text {. }
$$

Let $B=B(Z)$ be the Blaschke product for a zero sequence $Z=\left\{z_{n}\right\}$ composed of elements of $A$. By (6), (3) and the assumption on $F$, we have

$$
\|B F\|^{2}=\|F\|^{2}+\pi \sum_{n} P_{z_{n}}\left[\left|F\left(e^{i t}\right)\right|^{2}\right]<\infty,
$$

so the function $B F$, whose zero sequence is precisely $Z$, belongs to $\mathscr{D}$. Therefore, it is enough to construct the outer function $F \in \mathscr{D}$ satisfying (22). This can be done using the above mentioned result in [10], but we propose an ele- 
mentary approach, which do not depend on the deep methods of [10].

Let $f(t)=\operatorname{dist}\left(\tilde{A}, e^{i t}\right), t \in(-\pi, \pi]$. By the assumption on $A, f$ is log-integrable. Also, it is clear that $f$ is Lipschitz continuous :

$$
|f(t)-f(s)| \leqq|t-s|, \quad t, s \in(-\pi, \pi] .
$$

We claim that there are a continuous function $h$ on $(-\pi, \pi]$ and positive constants $c_{0}, c_{1}, c_{2}$, such that on the set $\{t \in(-\pi, \pi]: f(t)>0\}$ we have $c_{0} f(t) \leqq h(t)$ $\leqq f(t)$ and $h^{(n)}(t) \leqq c_{n} f(t)^{1-n}, n=1,2$. The construction of $h$ is left to the interested reader. Let $F$ be the outer function given by $\left|F\left(e^{i t}\right)\right|=\left|h\left(e^{i t}\right)\right|, t \in(-\pi, \pi]$. Lemma 5 with $g(t)=\left|F\left(e^{i t}\right)\right|^{2} \in C^{1+\varepsilon}(\boldsymbol{T})$ (any $0<\varepsilon<1$ ), yields $P_{z}\left[\left|F\left(e^{i t}\right)\right|^{2}\right]=$ $O(1-|z|)$ on $\left\{z=r e^{\imath \varphi} \in \Delta: 1-r \geqq\left|F\left(e^{\imath \varphi}\right)\right|^{2}\right\} \supset \widetilde{A} \supset A$. Finally by $(6)$ and $g \in C^{1+\varepsilon}(\boldsymbol{T})$, we have

$$
\begin{aligned}
\|F\|^{2} & \leqq \frac{1}{16 \pi} \int_{0}^{\pi} d t \int_{-\pi}^{\pi} d x|\log g(x)||2 g(x)-g(x+t)-g(x-t)| \sin ^{-2} \frac{t}{2} \\
& \leqq \frac{1}{16 \pi} \int_{0}^{\pi} d t \int_{-\pi}^{\pi} d x|\log g(x)| C t^{1+\varepsilon} \sin ^{-2} \frac{t}{2}<\infty,
\end{aligned}
$$

with a constant $C<\infty$, and so $F \in \mathscr{D}$. This completes the proof.

\section{Proof of Theorem 2}

The necessary condition (8) is much weaker than (9). To see this we note, that another condition

$$
\Sigma\left(1-\left|z_{n}\right|\right)^{1 / 2}<\infty
$$

implies (8) (we leave the verification to the reader). At the same time, as shown in Introduction, (9) is the weakest sufficient condition for moduli of zero sequences for $\mathscr{D}$. Theorem 2 is another precise formulation of this observation.

We need the following version of the Borel-Cantelli lemma given in [1].

LEMMA 6. If $\left\{A_{n}\right\}$ is a sequence of measurable subsets of some probability space $(\mathscr{X}, P)$ such that $\Sigma P\left(A_{n}\right)=\infty$ and

$$
\liminf _{n \rightarrow \infty} \frac{\sum_{j, k \leq n} P\left(A_{j} \cap A_{k}\right)}{\left[\sum_{k \leqq n} P\left(A_{k}\right)\right]^{2}} \leqq 1
$$

then $P\left(\lim \sup _{n \rightarrow \infty} A_{n}\right)=1$.

As usual, we write $\lim \sup _{n \rightarrow \infty} A_{n}$ for $\bigcap_{m=1}^{\infty} \bigcup_{n=m}^{\infty} A_{n}$.

Proof of Theorem 2. The first part of the theorem is the result of Shapiro and Shields in [9] discussed in Introduction. We now turn to the second part of the theorem. For $\theta \in(-\pi, \pi]$ define 


$$
\mathcal{E}(\theta)=\left\{r e^{\imath \varphi} \in \Delta: 1-r>\exp \left\{-\frac{1}{|\varphi-\theta|}\right\}\right\} .
$$

This region makes exponential contact with $\boldsymbol{T}$ at $e^{i \theta}$. It was proved in [6] that for every $F \in \mathscr{D}, H(\theta)=\lim F(z)$ exists as $z \rightarrow e^{i \theta}$ within $\mathcal{E}(\theta)$ for almost all $\theta \in$ $(-\pi, \pi]$. In particular, for $F \neq \equiv$, the set $\{\theta \in(-\pi, \pi]: H(\theta)=0\}$ is of zero Lebesgue measure. Let $\left\{r_{n}\right\}$ be a sequence with elements in $(0,1)$ for which

$$
\sum_{n=1}^{\infty} \frac{1}{\log _{\frac{1-r_{n}}{1-r}}}=\infty .
$$

Let $\left\{\Theta_{n}\right\}$ be a sequence of independent random variables on some probability space $(\Omega, \mu)$, uniformly distributed on $(-\pi, \pi\rfloor$. For $n=1,2, \cdots$, define $Z_{n}=$ $r_{n} e^{i \theta_{n}}$. Also, let $I_{n}$ be the interval on $(-\pi, \pi] \sim \boldsymbol{T}$ with the length $1 / \log \left(1 /\left(1-r_{n}\right)\right)$, and the center 0 , and $A_{n}=\left(I_{n}+\Theta_{n}\right) \bmod 2 \pi$ be the random interval of the same lenght and with the center $\Theta_{n}$. Note that for every $\varphi \in A_{n}$, we have $Z_{n} \in \mathcal{E}(\varphi)$. To prove that almost surely on $\Omega$ (a.s.), $\left\{Z_{n}\right\}$ is not a zero sequence for $\mathscr{D}$, we only need to show that $\left|\lim \sup _{n \rightarrow \infty} A_{n}\right|>0$ a.s., Indeed, for every $F \in \mathscr{D}$ having $\left\{Z_{n}\right\}$ as the zero sequence, the function $H$, defined above, is equal to zero a. e. on $\lim \sup _{n \rightarrow \infty} A_{n} \subset(-\pi, \pi]$.

In what follows we write $P$ for the normalized Lebesgue measure on $(-\pi, \pi]$. Let $E$ denote the expectation with respect to the probabilistic measure $\mu$ on $\Omega$. By Fubini's theorem we have $E P\left(A_{j} \cap A_{k}\right)=P\left(I_{\jmath}\right) P\left(I_{k}\right), \jmath \neq k$, and by Fatou's lemma and (25)

$$
\begin{aligned}
E & \liminf _{n \rightarrow \infty} \frac{\sum_{\jmath, k \leqq n} P\left(A_{j} \cap A_{k}\right)}{\left[\sum_{k \leqq n} P\left(A_{k}\right)\right]^{2}} \leqq \liminf _{n \rightarrow \infty} E \frac{\sum_{\jmath, k \leqq n} P\left(A_{j} \cap A_{k}\right)}{\left[\sum_{k \leqq n} P\left(A_{k}\right)\right]^{2}} \\
= & \liminf _{n \rightarrow \infty} \frac{\sum_{\jmath, k \leqq n} E P\left(A_{j} \cap A_{k}\right)}{\left[\sum_{k \leqq n} P\left(I_{k}\right)\right]^{2}}=\liminf _{n \rightarrow \infty} \frac{\sum_{\jmath \neq k} P\left(I_{\jmath}\right) P\left(I_{k}\right)+\sum_{k \leqq n} P\left(I_{k}\right)}{\left[\sum_{k \leqq n} P\left(I_{k}\right)\right]^{2}}=1 .
\end{aligned}
$$

This yields that (23) holds on a set $\mathscr{B} \subset \Omega$ of positive probability. By the zeroone law, $\mu(\mathscr{B})=1$. We have $P\left(\lim \sup _{n \rightarrow \infty} A_{n}\right)=1$ a.s. and the proof is complete.

\section{REFERENCES}

[1] P. Billingsley, Probability and Measure, John Wiley \& Sons, Inc., New YorkChichester-Brisbane-Toronto, 1979.

[2] L. CARLESON, A representation formula for the Dirichlet integral, Math. Z., 73 (1960), 399-400.

[3] J.G. Caughran, Two results concerning the zeros of functions with finite Dirichlet integral. Canad. J. Math., 21 (1969), 312-316.

[4] J.G. CAUGHRAN, Zeros of analytic functions with infinitely differentiable boundary values, Proc. Amer. Math. Soc., 24 (1970), 700-704.

[5] D. J. CAVENY AND W.P. Novinger, Boundary zeros of functions with derivative in $H^{p}$, Proc. Amer. Math. Soc., 25 (1970), 776-780. 
[6] A. NAGEL, W. Rudin AND J.H. Shapiro, Tangential boundary behaviour of function in Dirichlet-type spaces, Ann. of Math., 116 (1982), 331-360.

[7] J.D. Nelson, A characterisation of zero sets for $A^{\infty}$, Michigan Math. J., 18 (1971), 141-147.

[8] W.P. NovingER, Holomorphic functions with infinitely differentiable boundary values, Illinois J. Math., 15 (1971), 80-90.

[9] H.S. Shapiro And A.L. Shields, On the zeros of functions with finite Dirichlet integral and some related function spaces, Math. Z., 80 (1962), 217-229.

[10] B. A. TAYlor and D. L. Williams, Zeros of Lipschitz functions analytic in the unit disc, Michigan Math. J., 18 (1971), 129-139.

[11] S. A. Vinogradov and N.A. Shirokov, Zeros of analytic functions with derivative in $H^{1}$, Zapiski, 30 (1972), 154-157, in Russian.

[12] J.H. Wells, On the zeros of functions with derivatives in $H^{1}$ and $H^{\infty}$, Canad. J. Math., 22 (1970), 342-347.

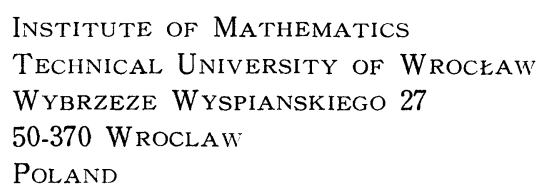

